Extra View

\title{
Excitotoxic insults lead to peroxiredoxin hyperoxidation
}

\author{
Frédéric Léveillé, Francesc X. Soriano, Sofia Papadia and Giles E. Hardingham* \\ Center for Integrative Physiology; University of Edinburgh; Edinburgh, UK
}

Key words: peroxiredoxin, NMDA receptor, excitotoxicity, oxidative stress, synaptic activity, sulfiredoxin, stroke

Post-mitotic neurons must have strong antioxidant defenses to survive the lifespan of the organism. We recently showed that neuronal antioxidant defenses are boosted by synaptic activity. Elevated synaptic activity, acting via the N-methyl-D-aspartate (NMDA) receptor, enhances thioredoxin activity, facilitates the reduction of hyperoxidized peroxiredoxins, and promotes resistance to oxidative stress. In contrast, blockade of spontaneous synaptic NMDA receptor activity renders neurons highly sensitive to hyperoxidation of peroxiredoxins by oxidative insults. These NMDA receptor-dependent effects are mediated in part by a coordinated program of gene expression changes centered on the thioredoxin-peroxiredoxin system, a thiol-based enzymatic system which is an important reducer of oxidative stressors such as hydroperoxides. We show here that while too little glutamatergic activity can render neurons vulnerable to peroxiredoxin hyperoxidation, so can too much. Exposure of neurons to toxic concentrations of glutamate, activating both synaptic and extrasynaptic NMDA receptors, acutely induces peroxiredoxin hyperoxidation. Thus, the effect of NMDA receptor activity on the activity of neuronal peroxiredoxins follows the classical U-shaped dose response curve.

\section{Introduction}

Correct redox regulation is essential in all cells, especially in post-mitotic cells such as neurons where harmful oxidative damage can accumulate. Oxidative damage and stress occurs when there is an imbalance between production of reactive oxygen species (ROS) and the cell's capacity to neutralize them through its intrinsic antioxidant defenses. Neurons are particularly susceptible to oxidative damage due to high levels of ROS production (through respiration and metabolism) and relatively low levels of certain antioxidant enzymes, particularly catalase. ${ }^{1,2}$ Oxidative damage is implicated in the pathogenesis of several neurodegenerative diseases as well as acute cerebrovascular disorders. ${ }^{1,2}$ Appropriate

*Correspondence to: Giles E. Hardingham; Centre for Integrative Physiology; University of Edinburgh; Edinburgh EH8 9XD UK; Tel.: +44.131.6507961; Fax: +44.131.6506527; Email: Giles.Hardingham@ed.ac.uk

Submitted: 02/24/09; Revised: 02/26/09; Accepted: 02/26/09

Previously published online as an Oxidative Medicine and Cellular Longevity E-publication:

http://www.landesbioscience.com/journals/oximed/article/8300 redox balance depends on the activity of antioxidant systems. Key among these are the thiol reducing systems based round thioredoxin and glutathione respectively, which are important reducers of many oxidative stressors such as peroxides. ${ }^{2,3}$ The principle source of peroxide is from spontaneous and superoxide dismutase (SOD)-catalysed dismutation of superoxide generated in active mitochondria. However, other sources of peroxides exist, including products of metabolic pathways involving oxidases/oxygenases (e.g., monoamine oxidase).

We recently showed that the vulnerability of neurons to oxidative death triggered by exposure to hydrogen peroxide was regulated by synaptic activity acting via $\mathrm{N}$-methyl-D-aspartate (NMDA) receptor (NMDAR) signaling. ${ }^{4}$ Neurons that were experiencing (or had recently experienced) higher levels of synaptic NMDAR activity were far more likely to withstand the oxidative insult than electrically quiet neurons. Accumulation of reactive oxygen species following an oxidative insult was significantly lower in active neurons than inactive ones. Neurons experiencing complete NMDAR blockade were highly vulnerable to peroxideinduced apoptosis in vitro, and NMDAR blockade in vivo promoted neuronal apoptosis associated with oxidative damage. It is not completely clear why synaptic activity, acting via $\mathrm{Ca}^{2+}$ signaling, should act to boost antioxidant defences. One possibility is to protect against increased ROS generation associated with high energy demand. Synaptic activity and activity-dependent plasticity place high energy demands on a neuron which must be largely met through oxidative phosphorylation, a process that generates ROS. Thus, active neurons would ordinarily need to have stronger antioxidant defenses than inactive ones in order to maintain the correct redox balance. However, synaptic activity is clearly strengthening antioxidant defenses above and beyond that required to cope with increased metabolic activity, since active neurons become resistant to additional exogenous insults. ${ }^{4}$

Investigations into the mechanism behind this revealed that synaptic activity exerted a number of changes to the thioredoxinperoxiredoxin system which contributed to the activity-dependent protection. ${ }^{4}$ Synaptic activity enhanced thioredoxin activity and facilitated the reduction of hyperoxidized peroxiredoxins, an important class of antioxidant enzymes. These changes were mediated by a coordinated program of gene expression changes. Synaptic NMDAR activity triggered the transcriptional suppression of the thioredoxin inhibitor Txnip, a protein known to 


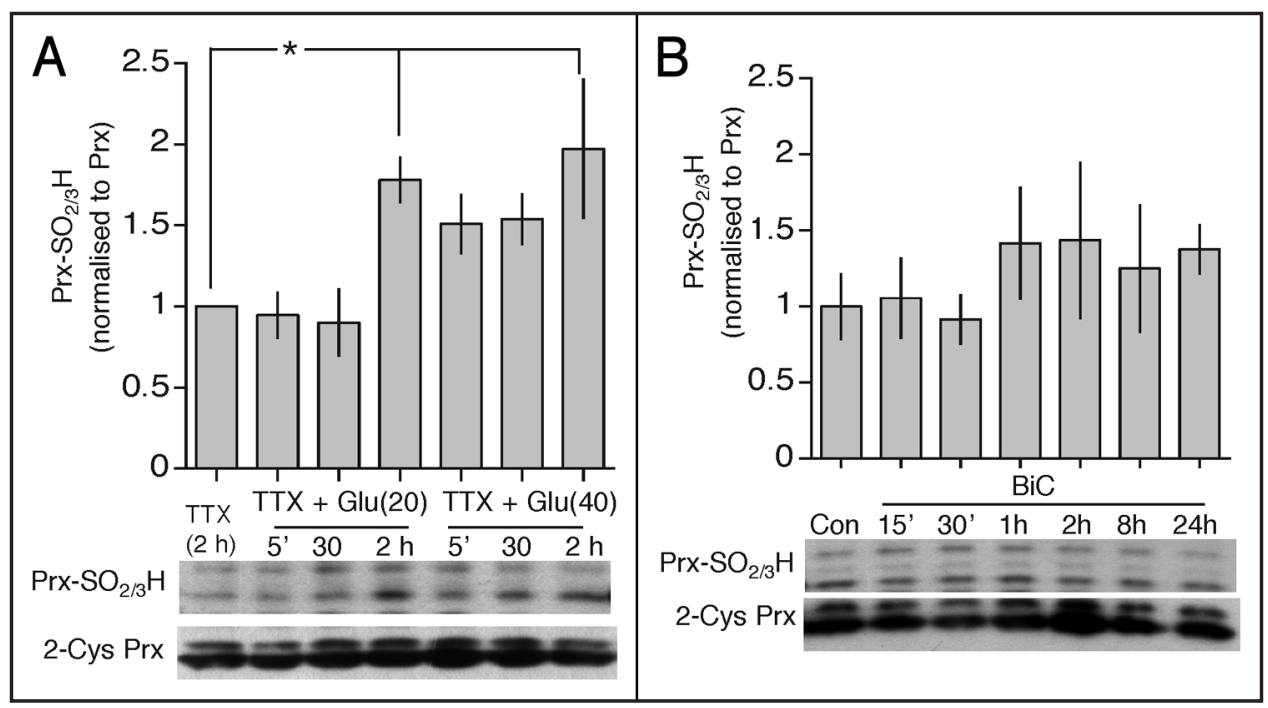

Figure 1. Excitotoxic glutamatergic activity promotes hyperoxidation of peroxiredoxins. (A and B) Lower: Example western analysis of peroxiredoxin (Prx) hyperoxidation in neurons treated as indicated, using an anti-PrxSO ${ }_{2 / 3} \mathrm{H}$ specific antibody. Upper: densitometric analysis of $\mathrm{PrxSO}_{2 / 3} \mathrm{H}$ band intensity of Prx II, normalized to the appropriate Prx II band. ${ }^{*} p<0.05$ compared to control (1-way Anova followed by Fisher's LSD test, $n=5$ ).

enhance oxidative stress. Furthermore, enhanced reduction of hyperoxidized peroxiredoxins was associated with transcriptional induction of two genes, sulfiredoxin and sestrin 2, whose products are reported to mediate this reaction. We did not address which of sestrin 2 or sulfiredoxin was responsible for reducing peroxiredoxin hyperoxidation. However, we recently showed that specific induction of sulfiredoxin is sufficient to prevent peroxiredoxin hyperoxidation in neurons. ${ }^{5}$ In any case, induction of one (or both) of these genes cooperated with the suppression of Txnip in boosting antioxidant defenses.

The effect of synaptic NMDAR activity in promoting the reduction of hyperoxidized peroxiredoxins may at first glance appear to be at odds with other studies that report the induction of oxidative stress and free radical production by NMDAR activity. For example, NMDAR activity has been shown to induce superoxide production using electron spin resonance detection. ${ }^{6}$ Indeed, agonists of all ionotropic glutamate receptors, as well as glutamate itself can induce rapid ROS generation. ${ }^{7,8}$ However, the ROS-generating capacity of agonists of ionotropic glutamate receptors are typically studied in the context of excitotoxic levels of agonists. Increased free radical production is likely a result of consequences of excessive $\mathrm{Ca}^{2+}$ influx, such as intracellular $\mathrm{Ca}^{2+}$ deregulation or mitochondrial dysfunction as well as other processes such as strong activation of nitric oxide production. ${ }^{9} 10$ In contrast, our stimulation paradigms used to promote synaptic activity are not toxic, quite the reverse-they are neuroprotective. ${ }^{11-13}$ Moreover, they do not induce mitochondrial dysfunction. ${ }^{11}$ The response of neurons to NMDAR activity follows an inverted U-shaped, or bell shaped curve, i.e., too much or too little can be potentially harmful. ${ }^{14,15}$ The studies which report ROS generation are generally concerned with the effect of high toxic levels of NMDAR activity, while we are looking at the central part of the curve where modest levels of NMDAR activity are protective.

\section{Results}

To contrast the effects of synaptic activity with those of toxic doses of glutamate, we have studied peroxiredoxin hyperoxidation in response to excitotoxic concentrations of glutamate (20 and $40 \mu \mathrm{M})$. Glutamate was added in the presence of tetrodotoxin to prevent preferential activation of synaptic NMDA receptors (which can happen in its absence ${ }^{16}$ ). We observed a significant elevation of levels of peroxiredoxin hyperoxidation at two hours post-treatment (Fig. 1A), indicative of oxidative stress. In contrast, stimulating synaptic activity using an established model (bicuculline plus 4-aminopyridine $(\mathrm{BiC} / 4-\mathrm{AP})^{13,17}$ ) triggers no peroxiredoxin hyperoxidation at any time point observed (Fig. 1B). This emphasizes the difference between the two stimulation paradigms.

It is worth noting that the difference between these two stimulation paradigms is not simply due to degree of NMDA receptor activity. If this was true then exposure of neurons to lower doses of glutamate (e.g., 5 and $10 \mu \mathrm{M}$ ) would trigger the same effects as $\mathrm{BiC} / 4-\mathrm{AP}$ treatment. However, these stimuli do not trigger the same anti-oxidative effects: reduction of peroxiredoxin hyperoxidation or sulfiredoxin/sestrin 2 upregulation is not promoted, nor is protection against oxidative stress. ${ }^{4}$ The key difference is that the strong antioxidative signaling described in ${ }^{4}$ relies on the trans-synaptic activation of synaptic NMDA receptors. Chronic activation of all (synaptic and extrasynaptic NMDA receptors) is unable to recapitulate trans-synaptically evoked signaling, regardless of dose. It is currently unclear how synaptic and extrasynaptic NMDARs would be coupled to different signaling pathways in the context of the effects described here. It could be that they are coupled differently (either physically or functionally) as a result of their differing location. Another contributing factor could be the way in which these distinct pools are activated: brief saturating activation in the case of trans-synaptic activation of synaptic NMDARs vs. chronic 
low level activation of extrasynaptic NMDARs by bath/ambient glutamate. Differences in the properties of intracellular $\mathrm{Ca}^{2+}$ transients evoked by these different stimuli could differentially affect signaling, even if the overall $\mathrm{Ca}^{2+}$ load were similar (reviewed in ref. 18).

The observation that exogenous glutamate exposure induced hyperoxidation of peroxiredoxins is interesting in view of the fact that this event also occurs in vivo following an excitotoxic insult-namely ischemia followed by reperfusion. ${ }^{4}$ Thus, it is possible that ROS generation triggered by excessive glutamate build up following an ischemic episode contributes to neuronal damage through inactivation of peroxiredoxins, in concert with the many other damaging events that take place, such as stress-activated protein kinase activation, calpain activation, nitric oxide production as well as inflammatory events and ionic imbalance. ${ }^{19-24}$

\section{Materials and Methods}

Neuronal cultures and stimulation. Cortical rat neurons were cultured as described ${ }^{25}$ from E21 rats except that growth medium was supplemented with B27 (Invitrogen). Stimulations were done in both cases after a culturing period of 8-10 days during which cortical neurons develop a network of processes, express functional NMDA-type and AMPA/kainate-type glutamate receptors, and form synaptic contacts. Stimulations were performed after transferring neurons into defined medium lacking trophic support "TMo": 13 10\% MEM (Invitrogen), 90\% Salt-Glucose-Glycine (SGG) medium; ${ }^{26}$ (SGG: $114 \mathrm{mM} \mathrm{NaCl}, 0.219 \% \mathrm{NaHCO}_{3}$,

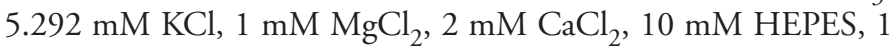
$\mathrm{mM}$ Glycine, $30 \mathrm{mM}$ Glucose, $0.5 \mathrm{mM}$ sodium pyruvate, $0.1 \%$ Phenol Red; osmolarity 325 mosm/l, hereafter TMo). Bursts of action potential firing were induced by treatment of neurons with $50 \mu \mathrm{M}$ bicuculline, and burst frequency was enhanced by addition of $250 \mu \mathrm{M} 4$-amino pyridine. ${ }^{27}$ Glutamate was applied at the indicated concentration in the presence of TTX ( $2 \mu \mathrm{M}$, Tocris).

Western blotting and antibodies. Total cell lysates were boiled at $100^{\circ} \mathrm{C}$ for $5 \mathrm{~min}$ in $1.5 \mathrm{x}$ sample buffer $(1.5 \mathrm{M}$ Tris $\mathrm{pH} 6.8$; Glycerol 15\%; SDS 3\%; $\beta$-mercaptoethanol 7.5\%; bromophenol blue $0.0375 \%)$. Gel electrophoresis and western blotting were performed using Xcell Surelock system (Invitrogen) using precast gradient gels (4-20\%) according to the manufacturer's instructions. The gels were blotted onto PVDF membranes, which were then blocked for one hour at room temperature with $5 \%(\mathrm{w} / \mathrm{v})$ non-fat dried milk in TBS with $0.1 \%$ Tween 20 . The membranes were then incubated at $4^{\circ} \mathrm{C}$ overnight with the primary antibodies diluted in blocking solution: 2-Cys Prx (1:500, Abcam), Prx-SO ${ }_{2 / 3} \mathrm{H}$ (1:1,000, Abcam). For visualisation of western blots, HRP-based secondary antibodies were used followed by chemiluminescent detection on Kodak X-Omat film. Western blots were analysed by digitally scanning the blots, followed by densitometric analysis (ImageJ). All analysis of $\operatorname{Prx}-\mathrm{SO}_{2 / 3} \mathrm{H}$ levels involved normalizing to the loading control: total 2-Cys Prx as determined using an anti-2-Cys Prx antibody. Blots were first probed with the anti-Prx- $-\mathrm{SO}_{2 / 3} \mathrm{H}$ antibody, then stripped and reprobed with anti-2-Cys Prx to determine loading.

\section{Concluding Remarks and Future Perspectives}

The detailed activity-dependent changes in gene expression which contribute to enhanced antioxidant defenses have hitherto focused on the thioredoxin system. ${ }^{4}$ However, these changes do not account for the full effects of synaptic activity, suggesting that other enzymic systems may also be targeted. It will be of interest to know how, or whether, synaptic activity boosts the capacity of the other key antioxidant system in neurons-that based on glutathione. Another outstanding issue is the degree to which protective changes in gene expression occur in vivo following an episode of NMDA receptor activity. Given that an episode of ischemia/reperfusion triggers hyperoxidation of peroxiredoxins, ${ }^{4}$ one might expect that a protective preconditioning episode reduces this. If this is indeed the case, this would beg the question as to whether the changes in gene expression, such as upregulation of the peroxiredoxin regenerating enzyme, sulfiredoxin, occur in response to preconditioning.

\section{Acknowledgements}

This work was funded by the Wellcome Trust and the Royal Society.

\section{References}

1. Mariani E, Polidori MC, Cherubini A, Mecocci P. Oxidative stress in brain aging, neurodegenerative and vascular diseases: An overview. J Chromatogr B Analyt Technol Biomed Life Sci 2005; 827:65-75.

2. Halliwell B. Oxidative stress and neurodegeneration: Where are we now? J Neurochem 2006; 97:1634-58.

3. Yoshida T, Nakamura H, Masutani H, Yodoi J. The involvement of thioredoxin and thioredoxin binding protein- 2 on cellular proliferation and aging process. Ann N Y Acad Sci 2005; 1055:1-12.

4. Papadia S, Soriano FX, Leveille F, Martel MA, Dakin KA, Hansen HH, et al. Synaptic NMDA receptor activity boosts intrinsic antioxidant defenses. Nat Neurosci 2008; 11:476-87.

5. Soriano FX, Leveille F, Papadia S, Higgins LG, Varley J, Baxter P, et al. Induction of sulfiredoxin expression and reduction of peroxiredoxin hyperoxidation by the neuroprotective Nrf2 activator ${ }^{3} \mathrm{H}$-1,2-dithiole-3-thione. J Neurochem 2008; 107:533-43.

6. Lafon-Cazal M, Pietri S, Culcasi M, Bockaert J. NMDA-dependent superoxide production and neurotoxicity. Nature 1993; 364:535-7.

7. Bondy SC, Lee DK. Oxidative stress induced by glutamate receptor agonists. Brain Res 1993; 610:229-33.

8. Reynolds IJ, Hastings TG. Glutamate induces the production of reactive oxygen species in cultured forebrain neurons following NMDA receptor activation. J Neurosci 1995; $15: 3318-27$

9. Arundine M, Tymianski M. Molecular mechanisms of glutamate-dependent neurodegeneration in ischemia and traumatic brain injury. Cell Mol Life Sci 2004; 61:657-68.

10. Nicholls DG, Johnson-Cadwell L, Vesce S, Jekabsons M, Yadava N. Bioenergetics of mitochondria in cultured neurons and their role in glutamate excitotoxicity. J Neurosci Res 2007; 85:3206-12.

11. Hardingham GE, Fukunaga Y, Bading H. Extrasynaptic NMDARs oppose synaptic NMDARs by triggering CREB shut-off and cell death pathways. Nat Neurosci 2002; 5:405-14.

12. Lee B, Butcher GQ, Hoyt KR, Impey S, Obrietan K. Activity-dependent neuroprotection and cAMP response element-binding protein (CREB): Kinase coupling, stimulus intensity, and temporal regulation of CREB phosphorylation at serine 133. J Neurosci 2005; 25:1137-48.

13. Papadia S, Stevenson P, Hardingham NR, Bading H, Hardingham GE. Nuclear $\mathrm{Ca}^{2+}$ and the cAMP response element-binding protein family mediate a late phase of activitydependent neuroprotection. J Neurosci 2005; 25:4279-87.

14. Lipton SA, Nakanishi N. Shakespeare in Love-With NMDA receptors? Nature Medicine 1999; 5:270-1

15. Papadia S, Hardingham GE. The Dichotomy of NMDA Receptor Signaling. Neuroscientist 2007.

16. Soriano FX, Papadia S, Hofmann F, Hardingham NR, Bading H, Hardingham GE Preconditioning doses of NMDA promote neuroprotection by enhancing neuronal excitability. J Neurosci 2006; 26:4509-18.

17. Mckenzie GJ, Stephenson P, Ward G, Papadia S, Bading H, Chawla S, et al. Nuclear $\mathrm{Ca}^{2+}$ and CaM kinase IV specify hormonal- and Notch-responsiveness. J Neurochem 2005; 93:171-85. 
18. Soriano FX, Hardingham GE. Compartmentalized NMDA receptor signalling to survival and death. J Physiol 2007; 584:381-7.

19. Aarts MM, Tymianski M. TRPM7 and ischemic CNS injury. Neuroscientist 2005; 11:116-23.

20. Bano D, Nicotera P. $\mathrm{Ca}^{2+}$ signals and neuronal death in brain ischemia. Stroke; a journal of cerebral circulation 2007; 38:674-6.

21. Soriano FX, Martel MA, Papadia S, Vaslin A, Baxter P, Rickman C, et al. Specific targeting of pro-death NMDA receptor signals with differing reliance on the NR2B PDZ ligand. J Neurosci 2008; 28:10696-710.

22. Besancon E, Guo S, Lok J, Tymianski M, Lo EH. Beyond NMDA and AMPA glutamate receptors: Emerging mechanisms for ionic imbalance and cell death in stroke. Trends Pharmacol Sci 2008; 29:268-75.

23. Endres M, Engelhardt B, Koistinaho J, Lindvall O, Meairs S, Mohr JP, et al. Improving outcome after stroke: Overcoming the translational roadblock. Cerebrovascular diseases (Basel, Switzerland) 2008; 25:268-78.

24. Amantea D, Nappi G, Bernardi G, Bagetta G, Corasaniti MT. Post-ischemic brain damage: pathophysiology and role of inflammatory mediators. Febs J 2009; 276:13-26.

25. Bading H, Greenberg ME. Stimulation of protein tyrosine phosphorylation by NMDA receptor activation. Science 1991; 253:912-4.

26. Bading H, Ginty DD, Greenberg ME. Regulation of gene expression in hippocampal neurons by distinct calcium signaling pathways. Science 1993; 260:181-6.

27. Hardingham GE, Arnold FJ, Bading H. Nuclear calcium signaling controls CREB mediated gene expression triggered by synaptic activity. Nat Neurosci 2001; 4:261-7. 


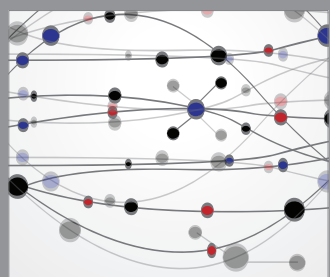

The Scientific World Journal
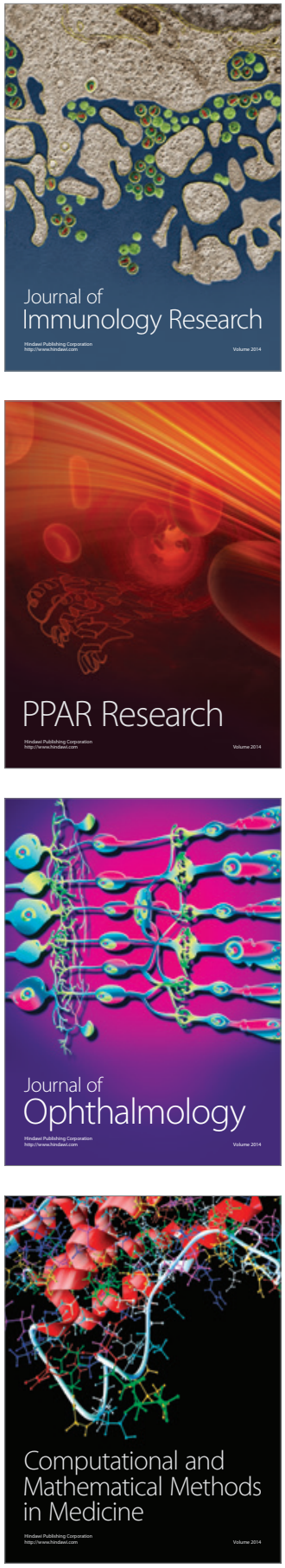

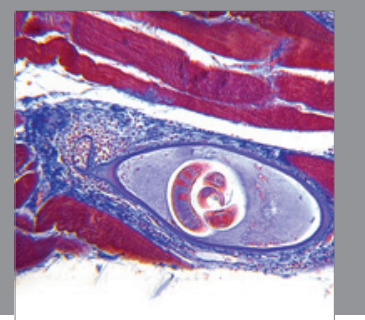

Gastroenterology

Research and Practice
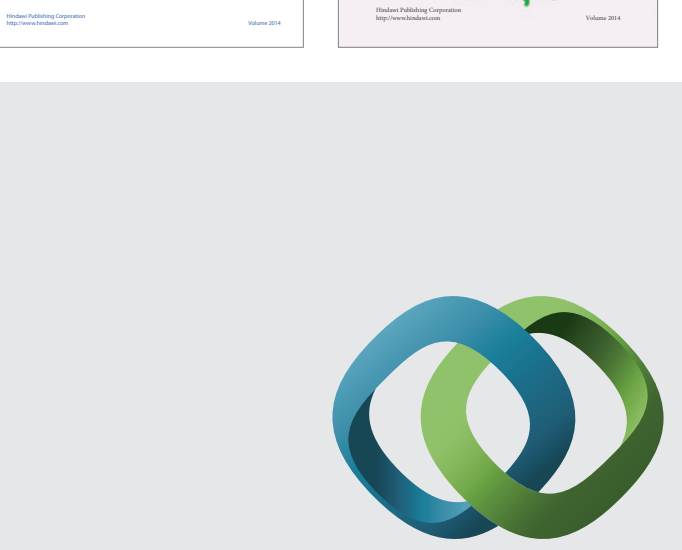

\section{Hindawi}

Submit your manuscripts at

http://www.hindawi.com
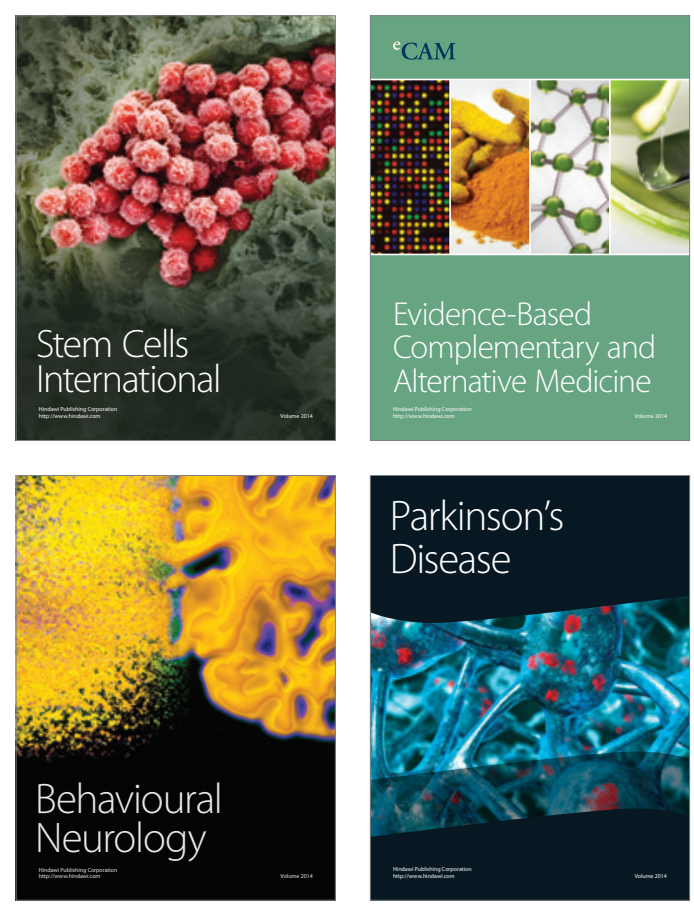

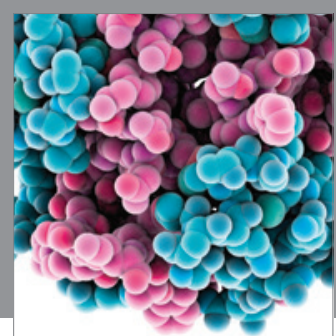

Journal of
Diabetes Research

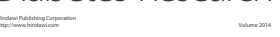

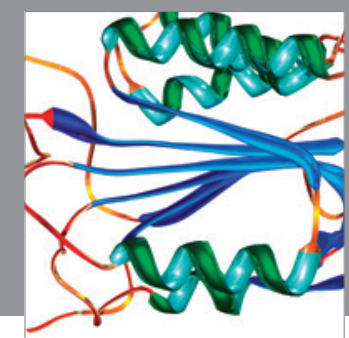

Disease Markers
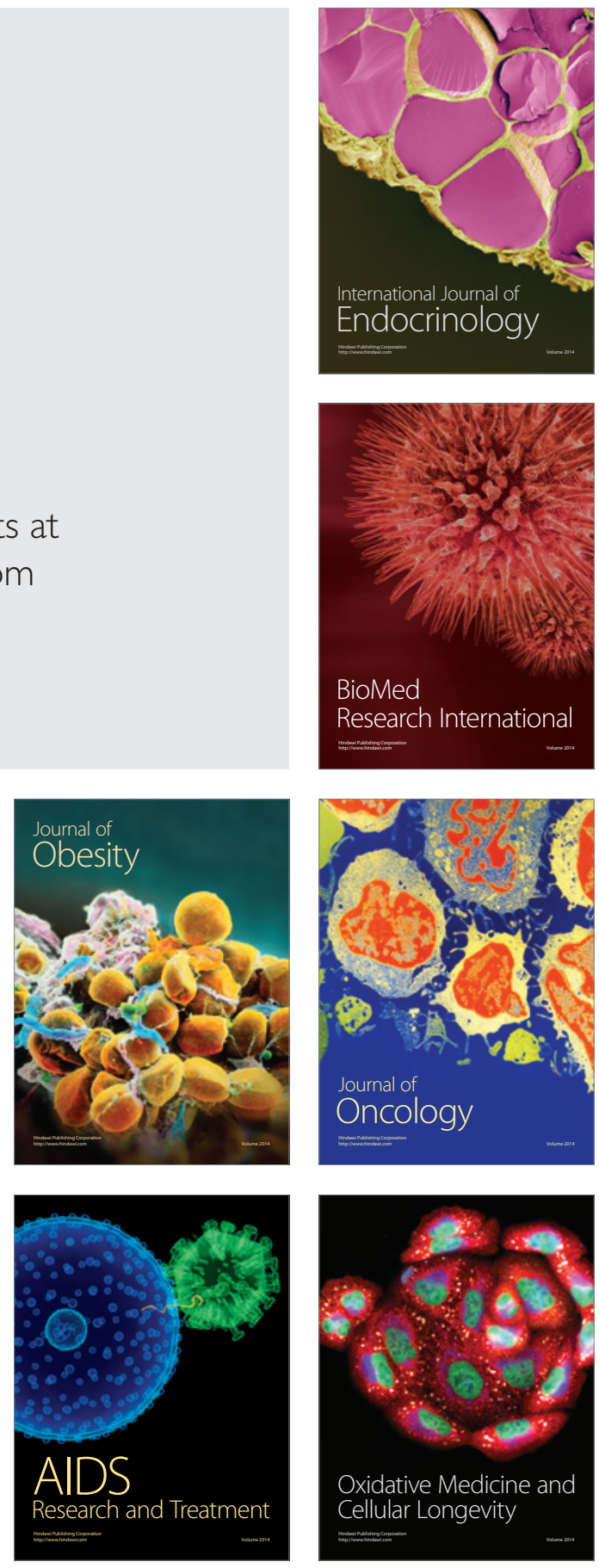